\title{
ON CAT (X\p)
}

\author{
JUAN JULIAN RIVADENEYRA PEREZ \\ Departamento de Matemáticas \\ Universidad Autonoma Metropolitana, Iztapalapa \\ A.P. 55-534; C.P. 09340 México D.F. México
}

(Received November 13, 1990 and in revised form February 28, 1991)

ABSTRACT. The Lusternik-Schnirelmann category of a PL-manifold does not increase if we delete a point of it. This is false in the CW-complex category.

KEY WORDS AND PHRASES. Lusternik-Schnirelmann category, categorical set, covering, CWcomplex, PL-manifold, cell, $n$-equivalence, universal covering. '1991 AMS SUBJECT CLASSIFICATION CODE. 55M30, 55S37.

\section{INTRODUCTION.}

The category (in the sense of Lusternik-Schnirelmann) of a topological space $X$, cat $(X)$, is the smallest number $k$ (if there is one) for which there exists an open covering $A_{1}, \ldots, A_{k}$ of $X$ such that every inclusion map $A_{i} \hookrightarrow X$ is null homotopic. One such covering is called an open categorical covering and every member of it is called an open categorical set.

To avoid trivialities and by simplicity, every space in the following will be supposed path connected.

Then a space $X$ is contractible if and only if it has category one and, since a suspension space $X=\sum Y$ is the union of two open cones we have that cat $(\mathrm{X}) \leq 2$ in this case. In particular if $S^{n}$ denotes the $n$-sphere then $\operatorname{cat}\left(S^{n}\right)=2$.

Now if $X$ is a retract of $Z$ and $\left\{A_{i}\right\}$ is an open categorical covering of $Z$ then $\left\{A_{i} \cap X\right\}$ is an open categorical covering of $X$, so that $\operatorname{cat}(X) \leq \operatorname{cat}(Z)$. Since $X$ can be embeded in $X \times Y$ as a retract we have that $\operatorname{cat}(X) \leq \operatorname{cat}(X \times Y)$.

It can be shown that if $X$ is a finite CW-complex we can replace 'open set' by 'closed subcomplex' in the definition of $\operatorname{cat}(X)$; moreover if $Y$ is another space of the same type then $\operatorname{cat}(X \times Y) \leq \operatorname{cat}(X)+\operatorname{cat}(Y)-1$. For these and other properties of this homotopy invariant we refer to the survey article of I.M. James [1].

Putting $Y=S^{1}$ in the previous inequalities involving $X \times Y$ we obtain that $\operatorname{cat}(X) \leq \operatorname{cat}\left(X \times S^{1}\right) \leq \operatorname{cat}(X)+1$, where $X$ is a finite CW-complex.

A very interesting, and classical, problem arising from the last inequalities is to determine when the equality is satisfied in the second of them. Singhof [2] and Montejano [3] have proved that if $X$ is a PL-compact manifold without boundary, then that equality is satisfied under certain stability conditions on the category. One of the steps of each proof is to embed $X$ as a retract of the complement of a ball in $X \times S^{1}$. Motivated by this we have the following generalization of the $\operatorname{cat}\left(X \times S^{1}\right)$-problem:

What is the category of $X \backslash p$, where $X$ is a compact manifold without boundary and $p$ is a point in it? 
In this paper we use obstruction theory and geometric arguments to prove that if $X$ is a PLmanifold then $\operatorname{cat}(X \backslash p) \leq \operatorname{cat}(X)$. Moreover we give examples of finite $\mathrm{CW}$-complexes for which $\operatorname{cat}(X)<\operatorname{cat}(X \backslash p)$.

2. ON CAT $(X \backslash p)$.

If $X$ is a finite CW-complex and $p$ is a point in $X$ then it is clear that $\operatorname{cat}(X) \leq \operatorname{cat}(X \backslash p)+1$ but in general it is not true that $\operatorname{cat}(X \backslash p) \leq \operatorname{cat}(X)$.

2.1 EXAMPLES. i) Let $S^{k}$ be the sphere of dimension $k$, and $\left(S^{k}\right)^{n}$ the cartesian product of $n$ copies of $S^{k}$. Then if $m<n$ let $X$ be the wedge of $\left(\left(S^{k}\right)^{m}, q\right)$ and $\left(C\left(S^{k}\right)^{n}, \widetilde{q}\right)$ where $C\left(S^{k}\right)^{n}$ is the unreduced cone over $\left(S^{k}\right)^{n}$ and $\widetilde{q}$ is a point in its base. Then $X$ and $\left(S^{k}\right)^{m}$ are homotopy equivalent, and if $p$ is the vertex of the cone we have that $X \backslash p$ has the same homotopy type as $\left(S^{k}\right)^{n} V\left(S^{k}\right)^{m}$, and

$$
m+1=\operatorname{cat}(X)<\operatorname{cat}(X \backslash p)=n+1
$$

Hence the difference $\operatorname{cat}(X \backslash p)-\operatorname{cat}(X)$ can be arbitrarily large and this fact is independent of connectedness.

ii) A less trivial example is obtained by capping a hole of the torus $T=S^{1} \times S^{1}$ as in the figure

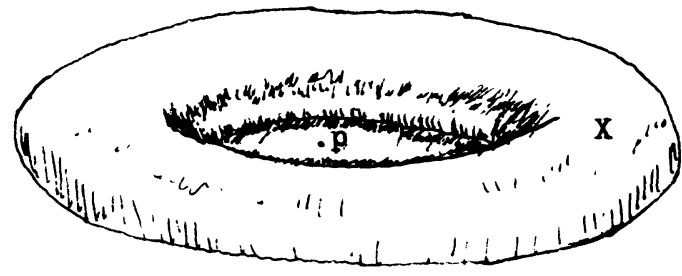

I. $e, X=T \cup_{j} D^{2}$ where $j: S^{1} \rightarrow T$ represents a generator of $\pi_{1}(T)$.

In this case $X$ and $X \backslash p$ have the same homotopy type as $S^{2} V S^{1}$ and $T$ respectively, and

$$
2=\operatorname{cat}(X)<\operatorname{cat}(X \backslash p)=3
$$

REMARK. At this point it is convenient to notice that cat $(X \backslash p)$ depends on the choice of $p$, for example if in 2.1.i) $\widetilde{p}$ is a point in $\left(S^{k}\right)^{m} \backslash(q)$ then cat $(X \backslash \widetilde{p})=m$, or if in 2.1.ii) $\widetilde{p}$ is a point in $T \backslash D$ then cat $(X \backslash \widetilde{p})=2$.

We shall prove now that if $X$ is a PL-manifold then $\operatorname{cat}(X \backslash p) \leq \operatorname{cat}(X)$. We will use the notation found in Spanier [4; chapter 8] and in Cohen [5].

THEOREM 2.2. Let $X$ be a PL-compact $n$-manifold without boundary. If $\operatorname{cat}(X) \geq 3$ and $p$ is a point in $X$ then $\operatorname{cat}(X \backslash p) \leq \operatorname{cat}(X)$.

For the proof we will need the following lemma.

LEMMA 2.3. Let $X$ be a PL-compact $n$-manifold without boundary. If $\operatorname{cat}(X)=k \geq 3$ then there exists an open $n$-cell $e$ and a categorical covering $A_{1}, \ldots, A_{k}$ of $X$ such that $A_{i} \downarrow B_{i}$ and $B d(e) \cong S^{n-1}$ is not a subset of $A_{i}$, for every $i$. Here the $B_{i}$ are subcomplexes of $X$ with dimension less than or equal to $n-2$ and $B d(e)$ denotes the topological boundary of $e$.

PROOF OF 2.2. We observe first that the spaces $X \backslash p$ and $X \backslash e$, where $p$ is a point and $e$ is an $n$-cell, are of the same homotopy type. Then it is enough to prove that $\operatorname{cat}(X \backslash e) \leq \operatorname{cat}(X)$ for some particular cell $e$. Let $A_{1}, \ldots, A_{k}$ and $e$ be as in the lemma. If $A$ denotes $A_{1}$ we will prove that $A \backslash e$ is categorical in $X \backslash e$. Suppose first that $X$ is simply connected. Since the pair $(X, X \backslash e)$ is $(n-1)$ connected we have a characteristic class $\theta$ in $H^{n}(X, X \backslash e ; Z)$ such that in the principal fibration induced by $\theta$ we have the following $n$-factorization of the inclusion map $i$. 


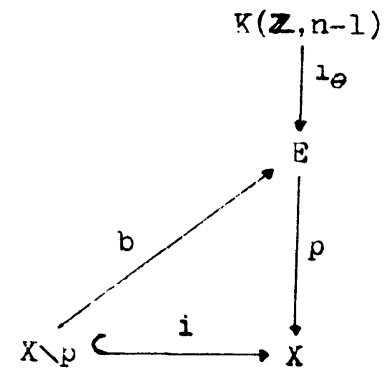

In particular $b$ is an $n$-equivalence. Let $j$ be the inclusion map of $A \backslash e$ in $X \backslash e$. Then $i \times j$ is null homotopic and there exists a lifting $j^{\prime}: A \backslash e \rightarrow K(\mathbb{Z}, n-1)$ such that $i_{\theta} \times j^{\prime}=b \times j$. Now if $\bar{e}$ is the closure of $e$ then $A \backslash e$ and $A \cap \bar{e}$ are subcomplexes of $X$, and using the Mayer-Vietoris theorem we have the following exact sequence

$$
H^{n-1}(A) \rightarrow H^{n-1}(A \backslash e) \oplus H^{n-1}(A \cap \bar{e}) \rightarrow H^{n-1}(A \cap \bar{e} \backslash e)
$$

But $A \cap \bar{e} \backslash e$ is a proper subset of $B d(e) \cong S^{n-1}$ and so $H^{n-1}(A \cap \bar{e} \backslash e)=0$. Moreover, since $A \approx B_{1}$, where $\operatorname{dim} B_{1} \leq n-2$, we have that $H^{n-1}(A)=0$ and from this we conclude that $H^{n-1}(A \backslash e)=0$. Then $i_{\theta} \times j^{\prime}=b \times j$ is null homotopic and this implies that $j$ is null homotopic because $A \backslash e$ has the homotopy type of a complex whose dimension does not exceed $n-1$, and because $b$ is an $n$-equivalence.

In the case that $X$ is not simply connected we consider the universal covering of $X, \widetilde{X} \stackrel{\widetilde{p}}{\rightarrow} X$. Then, by 5.11 of Massey [6], every component of $\widetilde{p}^{-1}(e)$ and $\widetilde{p}^{-1}(A)$ is homeomorphic to $e$ and $A$, respectively.

Using a similar argument as above we can prove that if $\widetilde{A}$ is a component of $\widetilde{p}^{-1}(A)$ then $\tilde{A} \backslash \tilde{p}^{-1}(e)$ is contractible in $\tilde{X} \backslash \widetilde{p}^{-1}(e)$ and therefore that $A \backslash e$ is contractible in $X \backslash e$. Since the same argument is valid for every $A_{i}$ we have that $A_{1} \backslash e, \ldots, A_{k} \backslash e$ is a categorical covering of $X \backslash e$. This concludes the proof of Theorem 2.2.

PROOF OF LEMMA 2.3. The first part is a particular case of Theorem 1 in Montejano [7]. For the second part we take a point $p$ in $X \backslash\left(A_{1} \cup \ldots \cup A_{k-1}\right)$ and a point $q$ in $X \backslash A_{k}$. Let $\mathrm{C}$ be a simple path between $p$ and $q$. Then if $e$ is a sufficiently small tubular neighborhood of $\mathfrak{C}$ (near $\mathrm{C}$ we have a metric) we have that $B d(e) \backslash A_{i}$ is not empty for every $i$. This concludes the proof of Lemma 2.3.

REMARK. For $\operatorname{cat}(X)=2$, since any compact manifold without boundary with category two is a homotopy sphere, the inequality in Theorem 2.2 is valid in dimensions in which the generalized Poincare conjecture is true.

The arguments in the proof of 2.2 can be exploited to give the following criterion, in terms of the generalized Hopf invariant in Bernstein-Hilton [8], for the increasing of the category.

THEOREM 2.4. Let $X$ be a simply connected $n$-manifold as in 2.2. Let $\Delta: \bar{e} \rightarrow X$ be a closed $n$-cell with $\Delta S_{S^{n-1}}$ an embedding. If $p$ is an interior point of $\Delta(\bar{e})$ then $\operatorname{cat}(X \backslash p)<\operatorname{cat}(X)$ if and only if the generalized Hopf invariant, $H(\partial \Delta)$, of $\partial \Delta=\Delta \mid S^{n-1}: S^{n-1} \rightarrow X \backslash \Delta(e)$, is not zero.

PROOF. Suppose that $\operatorname{cat}(X \backslash p)=\operatorname{cat}(X)=k$, then from the proof of 2.2 we have that the inclusion $X \backslash \Delta(e) \rightarrow X$ is $k$-primitive and by Corollary 3.18 of Bernstein-Hilton [8] we conclude that $H(\partial \Delta)=0$ which is a contradiction. The other part follows from Propositions 3.3 and 3.4 of Bernstein-Hilton [8]. This concludes the proof of Theorem 2.4. 
With the following remarks we complete our calculations of $\operatorname{cat}(X \backslash p)$.

REMARKS. i) If $X$ is a manifold with boundary $\partial X$ and $p$ is a point in $X \backslash \partial X$ then there is a closed $n$-cell $\bar{e}$ such that $p$ is in $e$ and the intersection $\bar{e} \cap \partial X$ is an $(n-1)$-cell. Then $X \backslash \bar{e} \uparrow X$ and $X \backslash p \approx X V S^{n-1}$, and we conclude that $\operatorname{cat}(X \backslash e)=\operatorname{cat}(X \backslash p)=\operatorname{cat}(X)$.

ii) Let $X$ be as in i). If $p$ is a point in $\partial X$ then $X \backslash p$ and $X$ are of the same homotopy type. Then $\operatorname{cat}(X)=\operatorname{cat}(X \backslash p)$.

iii) If $p$ and $q$ are points in a manifold without boundary $X$ then $X \backslash p$ is homeomorphic to $X \backslash q$, hence the number $\operatorname{cat}(X \backslash p)$ is independent of the choice of $p$ if $X$ is a manifold.

Finally, we remark that due to the irregular behavior of $\operatorname{cat}(X \backslash p)$ for $\mathrm{X}$ a $\mathrm{CW}$-complex, it is not clear what the relation between $\operatorname{cat}(A)$ and $\operatorname{cat}(X)$ should be, given only that $A$ is a subcomplex of $\boldsymbol{X}$.

ACKNOWLEDGEMENT. This paper was motivated by discussions with Monica Clapp and Luis Montejano.

\section{REFERENCES}

1. JAMES, I.M., On category in the sense of Lusternik-Schnirelmann. Topology 17 (1978) 331348.

2. SINGHOF, W., Minimal coverings of manifolds with balls. Manuscripta Math 29 (1979), 385415.

3. MONTEJANO, L., A quick proof of Singhof's $\operatorname{cat}\left(M \times S^{1}\right)=\operatorname{cat}(M)+1$ theorem. Manuscripta Math. 42 (1983) 49-52.

4. SPANIER, E., Algebraic Topology, New York, McGraw Hill, 1966.

5. COHEN, M.M., A Course in Simple-Homotopy Theory. Graduate Texts in Mathematics, Vol. 10, Springer-Verlag, New York, Heidelberg-Berlin, 1973.

6. MASSEY, W.S., Algebraic Topology: An Introduction. New York: Harcourt, Brace and World, 1967.

7. MONTEJANO, L., Categorical and contractible covers of polyhedra. Topology and its appl. $\underline{32}$ (1989) 251-266.

8. BERNSTEIN, I. and HILTON, P., Category and Generalized Hopf invariants. Illinois J. Math. 4 (1960) 437-451. 


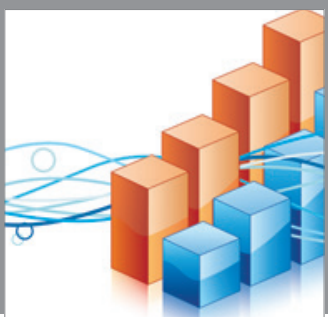

Advances in

Operations Research

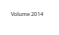

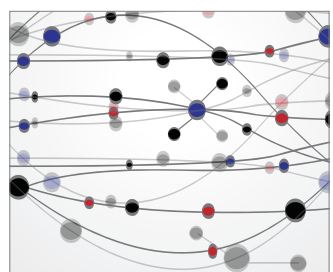

\section{The Scientific} World Journal
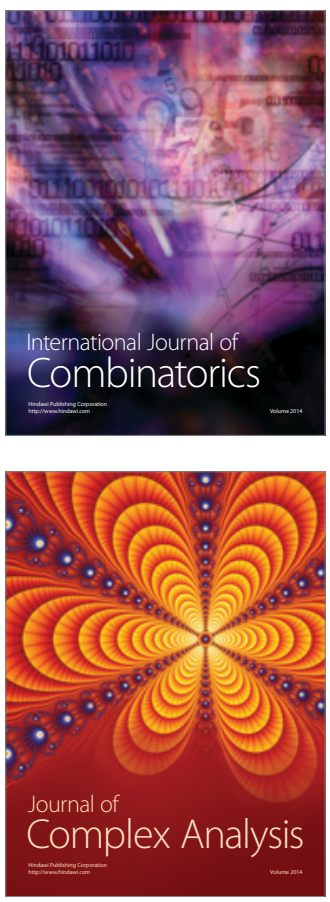

International Journal of

Mathematics and

Mathematical

Sciences
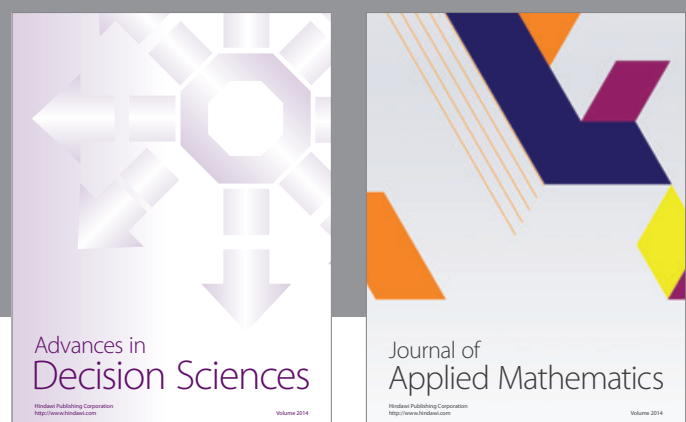

Journal of

Applied Mathematics
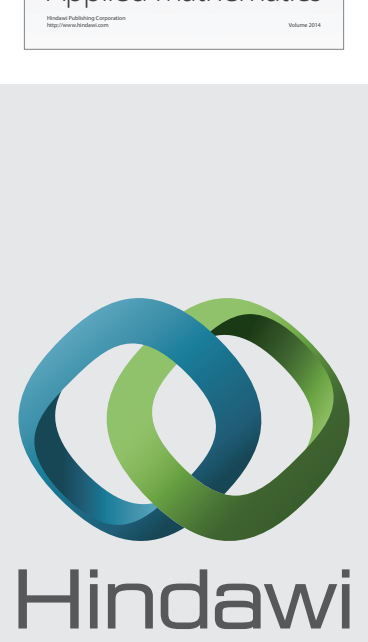

Submit your manuscripts at http://www.hindawi.com
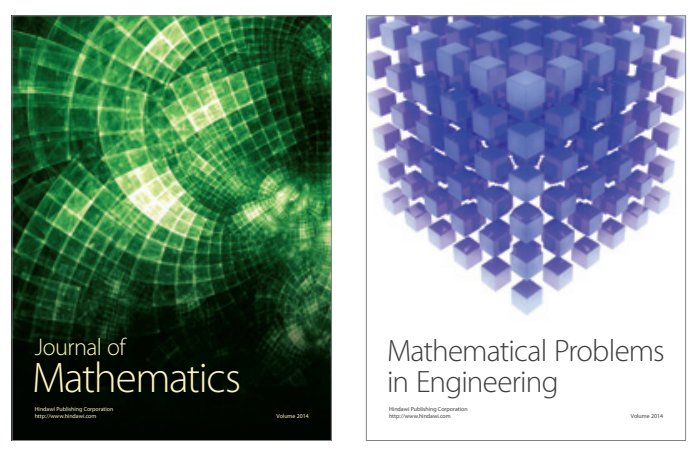

Mathematical Problems in Engineering
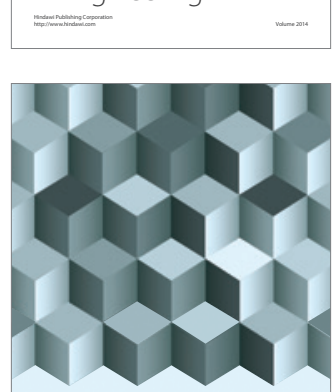

Journal of

Function Spaces
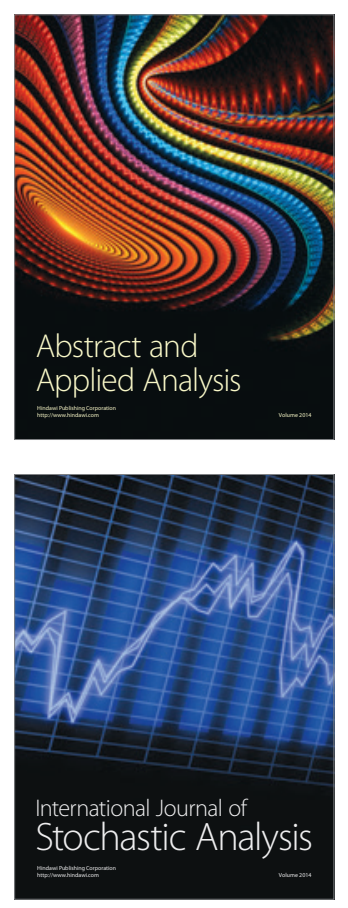

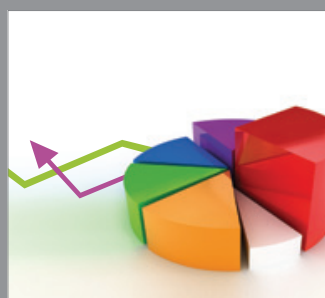

ournal of

Probability and Statistics

Promensencen
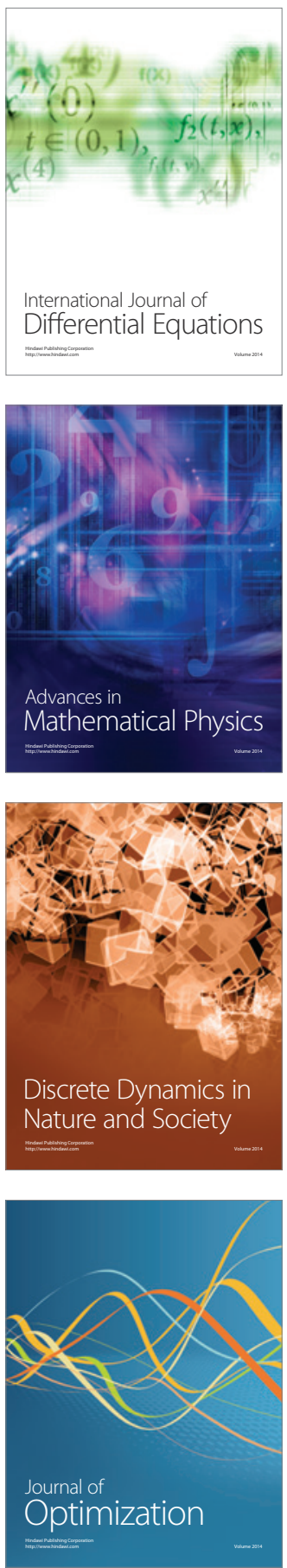